\title{
Modelling of spacecraft spin period during eclipse
}

\author{
E. Georgescu ${ }^{1}$, F. Plaschke ${ }^{2}$, U. Auster ${ }^{2}$, K.-H. Fornaçon ${ }^{2}$, and H. U. Frey ${ }^{3}$ \\ ${ }^{1}$ Max-Planck Institute for Solar System Research, Katlenburg-Lindau, Germany \\ ${ }^{2}$ Institute for Geophysics and Extraterrestrial Physics, Technical University Braunschweig, Germany \\ ${ }^{3}$ Space Sciences Laboratory, University of California, Berkeley, USA
}

Received: 18 November 2010 - Revised: 18 February 2011 - Accepted: 5 May 2011 - Published: 19 May 2011

\begin{abstract}
The majority of scientific satellites investigating the Earth magnetosphere are spin stabilized. The attitude information comes usually from a sun sensor and is missing in the umbra; hence, the accurate experimental determination of vector quantities is not possible during eclipses. The spin period of the spacecraft is generally not constant during these times because the moment of inertia changes due to heat dissipation. The temperature dependence of the moment of inertia for each spacecraft has a specific signature determined by its design and distribution of mass. We developed an "eclipse-spin" model for the spacecraft spin period behaviour using magnetic field vector measurements close to the Earth, where the magnetic field is dominated by the dipole field, and in the magnetospheric lobes, where the magnetic field direction is mostly constant. The modelled spin periods give us extraordinarily good results with accumulated phase deviations over one hour of less than 10 degrees. Using the eclipse spin model satellite experiments depending on correct spin phase information can deliver science data even during eclipses. Two applications for THEMIS B, one in the lobe and the other in the lunar wake, are presented.
\end{abstract}

Keywords. Magnetospheric physics (Planetary magnetospheres; Instruments and techniques) - Solar physics, astrophysics, and astronomy (Magnetic fields)

\section{Introduction}

The period of a spinning spacecraft is usually determined with a sun sensor. It depends on the moment of inertia of the spacecraft which in turn depends on the temperature of the spacecraft body. The temperature of the spacecraft is falling during eclipse and the spacecraft contracts diminishing its moment of inertia and hence the spin period until a

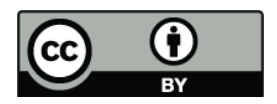

Correspondence to: E. Georgescu (georgescu@mps.mpg.de) final equilibrium is reached or the spacecraft exits the eclipse and heats up and expands again. The temperature dependence of the moment of inertia is mainly determined by the wire antennae and booms because of their extension and low thermal inertia. The spacecraft is an irregular object with non-homogeneous thermal properties and a theoretical calculation of the change of moment of inertia due to temperature change is difficult. Since the sun sensor doesn't deliver information during eclipses, the spin locked instruments and the vector measurements cannot work properly. We developed a method to determine the behaviour of the spacecraft spin period during eclipse using magnetic field measurements in the magnetosphere. The method requires eclipse measurements of the magnetic field in a region where either a model magnetic field is available or where the ambient magnetic field has a relatively constant direction. The model can subsequently be applied to reconstitute the spin period for eclipses outside the magnetosphere even when magnetic field measurements are not available.

\section{Description of the method}

The modelling of the spin during eclipse is based on the assumption that each spacecraft has a specific signature in the temperature dependence of its moment of inertia. The model intends to describe this signature analytically. The spin period can be determined from a magnetic field component in a rotating spacecraft system.

The method implies the following steps:

- determination of the spin period using a spinning component of the magnetic field in the spacecraft frame,

- correction of the spin period with the changes of the direction of the magnetic field in the spin plane determined from the IGRF magnetic field model and recording of this information into an eclipse data base, 

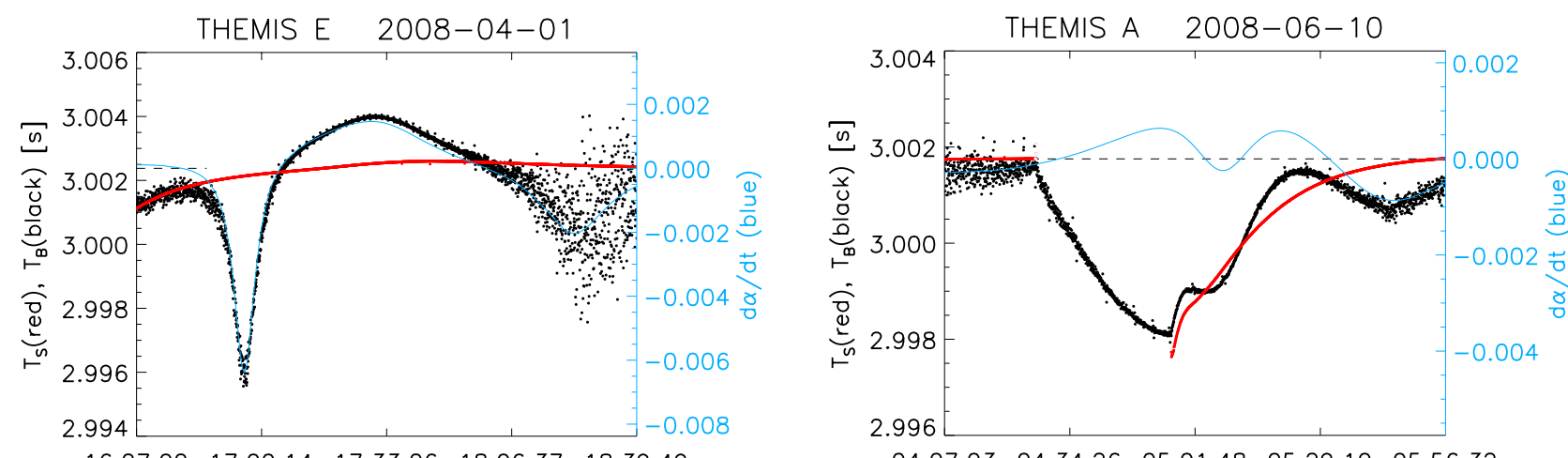

16:27:02 17:00:14 17:33:26 18:06:37 18:39:49
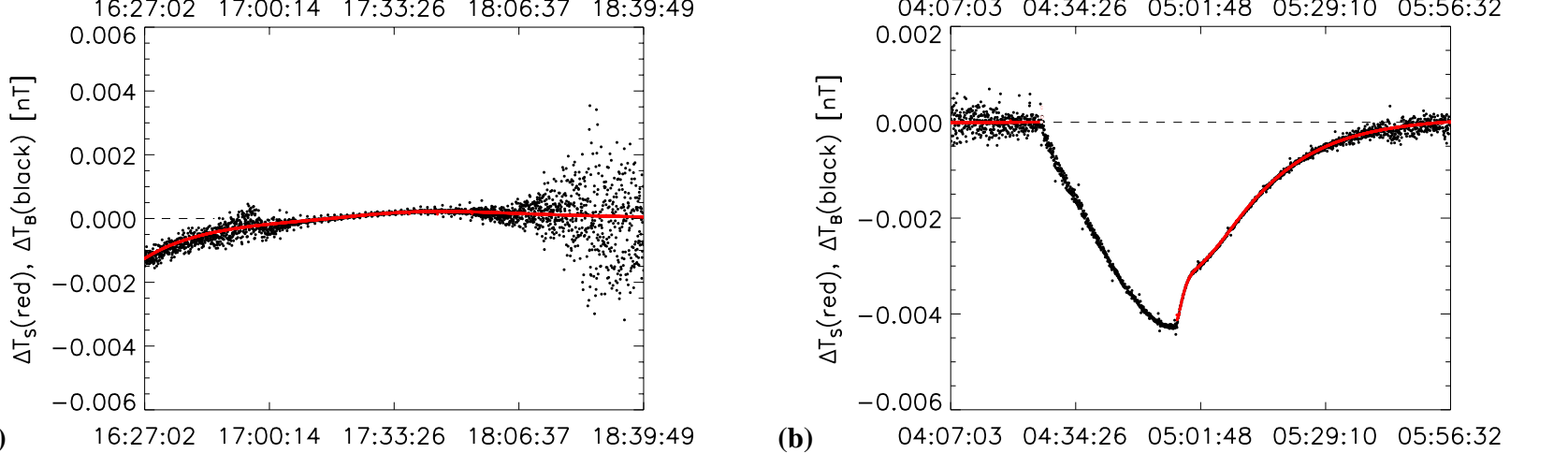

Fig. 1. Examples of spin behaviour for THEMIS. (a) no eclipse and (b) eclipse. Top panel: Sun sensor spin-period ( $T_{S}$ : red), FGM spin-period $\left(T_{B}\right.$ : black), Model magnetic field rotation $\left(d \alpha / d t\right.$ : blue). Lower panel: $\Delta T_{S}\left(\right.$ red), corrected FGM spin period $\left(\Delta T_{B c}:\right.$ black).

- superposed epoch analysis of the eclipse data and fit of an analytical expression to the data and

- checking the model with randomly selected eclipse data to determine the errors of the model.

The first two steps of the method were developed using CLUSTER data, but then applied systematically to two years of data collected by the five spacecraft of the THEMIS mission.

\subsection{Spin period determination from the magnetic field}

The magnetic field is measured both on CLUSTER and THEMIS spacecraft with flux-gate magnetometers (FGM); the magnetometers are described in detail in Balogh et al. (2001) and Auster et al. (2008). They are placed on booms situated in the spacecraft spin plane. In order to determine the spin period we used one of the spin-plane components of the calibrated magnetic field in the spacecraft frame. The magnetic field direction is assumed to be constant during a spin. The experimental measurement was decomposed into intervals of data containing "one period sine" curves by finding the zeros and taking windows slightly larger ( 2 measurement points outside the interval) than two subsequent crossings of the zero line in the same direction. A sine with linearly varying amplitude, which takes into account the change of the magnetic field magnitude during a spin, was fitted to each data interval.

$B_{\mathrm{FIT}}=\left(a_{0}+a_{1} t\right) \sin \left(\frac{2 \pi t}{a_{2}}-a_{3}\right)$

The " $t$ " variable is the time and $a_{i},(i=0-3)$ are the four parameters of the fit; the period $a_{2}$ of the sine function, named $T_{B}$ in the following, estimates the spin period of the spacecraft $(T)$. Since the data have different sampling rates and the spacecraft different rotation periods, the window lengths in seconds are also different. The method is applicable to any spinning spacecraft where the sampling rate of the magnetometer $(f s)$ is high enough that the number of measurement points per spin $(n=f s T)$ is larger than 4 , the number of the parameters in $B_{\text {FIT }}$. This condition is generally satisfied by most of the spinning spacecraft: for THEMIS $T \sim 3 \mathrm{~s}$ and the sampling rate is 4 or $8 \mathrm{~Hz}$ for the eclipse time periods resulting in at least 12 data points/spin; for CLUSTER $f_{s}=22$ or $67 \mathrm{~Hz}$ and $T \sim 4 \mathrm{~s}$. The accuracy is increasing with increasing number of measurement points.

\subsection{Correction of the experimental FGM spin period}

Figure 1 exemplifies the method for two time intervals for the THEMIS spacecraft one without eclipse (a) and one with eclipse (b). In the upper panels the FGM spin period $T_{B}$ (black points) and the sun sensor spin period $T_{S}$ (red) are overplotted. The two lower panels show the spin periods 
measured by the two instruments relative to the reference value $T_{S \text { ref: }}: \Delta T_{S}=T_{S}-T_{S \text { ref }}$ (red) and here in black are the corrected FGM spin periods $\Delta T_{B \mathrm{c}}=T_{B \mathrm{c}}-T_{S \text { ref }}$ by using the relative rotation of the magnetic field as described below. The reference value $T_{S \text { ref }}$, has been determined as the median of $T_{S}$ over a time interval at least $20 \mathrm{~min}$ prior to the eclipse in order to avoid penumbra effects.

The FGM-periods $\left(T_{B}\right)$ show a discrepancy when compared to the sun-sensor determination of the spin period $\left(T_{S}\right)$ in the intervals when both measurements are available. The discrepancy between $T_{B}$ and $T_{S}$ comes from the failure of our assumption concerning the magnetic field direction being constant during a spin. The assumption may be right in the lobes, but close to the Earth, the direction changes. These changes can be determined from the magnetic field model and may reach 10 degrees per spin for THEMIS $\left(T_{S} \sim 3 \mathrm{~s}\right)$.

The determined spin periods $T_{B}$ depend on the direction of rotation of the field relative to the rotation of the spacecraft. The time derivative of the clock angle in the spin plane is used to make the correction. The magnetic field computed in GSE, by using IGRF and Tsyganenko96, was transformed to a non-spinning spacecraft frame using the attitude. The spinplane components of the model field were spline-interpolated to the $T_{B}$ times. The clock angle $\alpha$, i.e. the angle of the projection of the magnetic field vector in the spin-plane, was computed as the arctangent of the ratio of the two spin-plane components in the non-spinning spacecraft frame. The corrected FGM spin periods have been checked against the sunsensor periods in time intervals without eclipse. The blue curve in the upper panel of Fig. 1 shows the derivative of the clock-angle $d \alpha / d t$ that is used to correct the FGM spin periods $T_{B \mathrm{c}}=T_{B}-d \alpha / d t$; now $T_{B \mathrm{c}}$ agrees fairly well with $T_{S}$. The bottom panel shows the plots of the differences to the reference spin: $\Delta T_{S}$ (red) and $\Delta T_{B \mathrm{c}}$. Depending on the region where the measurement is performed the spread of $T_{B \mathrm{c}}$ can be relatively large and/or the magnetic field model inaccurate. For the modelling of the spin behaviour we have to select regions relatively close to Earth, where the field is strong and stable. In the same time we need a large range of eclipse lengths to have a model describing all experimentally possible cases. For this we select the lobes where the field has an almost constant direction.

\subsection{Superposed epoch analysis of eclipse data and model fit}

A data base containing all eclipse data for the five THEMIS spacecraft from 2007 to 2009 has been built. One difficulty was related to the determination of the start and stop times of the eclipses due to the effect of penumbrae. Two methods have been investigated: one based on theoretical calculations using the orbit data and the second on experimental sun sensor data; the second proved to be more accurate.

A data file has been recorded for each eclipse where FGM data and model field data were available. The data file con-

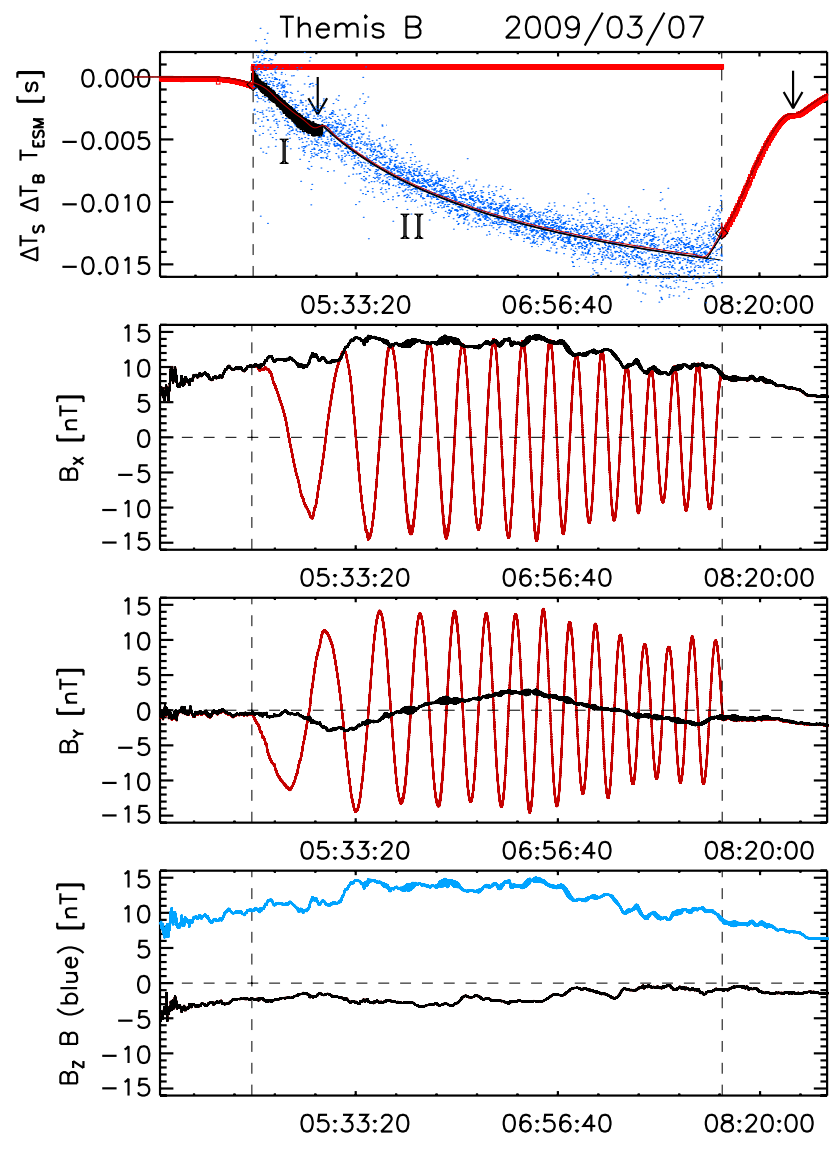

Fig. 2. Eclipse-spin model (ESM) and its application to despin magnetic field data. The eclipse time interval is delimited by the dashed lines. Top panel: difference of the spin period to the reference value before eclipse: $\Delta T_{S}$ (thick red line) from Sun sensor, $\Delta T_{B}$ (black and blue points) from magnetometer measurements and $T_{\mathrm{ESM}}$ (thin red line in the eclipse) eclipse spin model. I and II denote the interval of short and long eclipses and the arrow point to the shoulder in between. Lower 3 panels: magnetic field in spacecraft system; red: despun with constant $T_{S}$ and black: despun with $T_{\mathrm{ESM}}$. The magnitude of the field $B$ (blue) is shown in the bottom panel together with the component along the spin axis $B_{\mathrm{Z}}$ (black).

tained the time series of the sun-sensor periods $\left(T_{S}\right)$ and the corrected FGM periods $\left(T_{B \mathrm{c}}\right)$. Two reference values are needed to perform a superposed epoch analysis one in the timeline (EStart) and one in the spin period $\left(T_{\text {Sref }}\right.$ defined in Sect. 2.2). The eclipse start time, EStart, is defined as being the sun pulse time delivered by the sun sensor about $15 \mathrm{~s}$ before entering the eclipse. These values are subtracted from the time $(t)$ and from the spin period values $(T)$, respectively.

The numbers of files in the data base were 130, 20, 84, 177 and 152 for THEMIS A, B, C, D and E from a total of 231, $45,213,319$ and 324, respectively, because not every eclipse had FGM coverage. The majority of the eclipses are close to the Earth where the magnetic field strength is high and the duration of the eclipses is less than $30 \mathrm{~min}$. 
Figure 2 shows an interval of data of THEMIS B from 7 March 2009, where the spacecraft was in a long eclipse (delimited with the dotted vertical lines). The top panel contains relative spin periods to the reference value: the sun sensor measured relative periods $\Delta T_{S}$ (thick red line) that have a constant fake value in the eclipse and the values determined from FGM data ( $\Delta T_{B}$ blue data points). Overplotted in black starting at EStart are all $\Delta T_{B \mathrm{c}}$ for the close-toperigee eclipses in the year 2008.

All these FGM spin periods were used to define an empirical eclipse-spin model (ESM) which appears as a thin red line in the upper panel of Fig. 2.

The spread of the data points for these short $(<30 \mathrm{~min})$ eclipses is small compared to the blue dots for the long eclipse. Long eclipses are infrequent; they occur when the spacecraft are mostly far away in the tail where the magnetic field component in the spin-plane is small. Sometimes the field is disturbed by wave activity and hence $T_{B}$ cannot be determined with sufficient accuracy in order to be used for fitting a model.

The continuous decay of the spin period with time in the eclipse is interrupted with a relatively constant part that we name "shoulder" and mark with black arrows in the Figs. 2, 5 and 6 . A similar feature due to the same physical process appears during the warming up phase after re-entry in the sunlight and is specific to all THEMIS and CLUSTER spacecraft. This is determined by the spacecraft design, particularly the contraction and dynamics of the wire booms (Cherchas, 1971; Lai, 1979).

The fitting function was selected to reflect the physical processes supposed to produce the change in the spin period. The physics behind Eq. (2) is presented in Sect. 2.4.

The following function has been taken for the fit:

$T_{\mathrm{FIT}}=\left(\sqrt[3]{\frac{1}{\left(a_{0} t+a_{1}\right)^{2}}}-\sqrt[3]{\frac{1}{a_{1}^{2}}}\right) a_{2}+a_{3}$

where $a_{i},(i=0-3)$ are the free parameters and $t$ is the time in eclipse.

The fitting of the analytical function (2) is done in the two intervals I and II (top panel of Fig. 2) separated by the shoulder. The eclipse-spin model is given by a piecewise function (3) containing the results of the fit.

$T_{\mathrm{ESM}}= \begin{cases}T_{\mathrm{FIT}}^{\mathrm{I}} & t \leq t_{\mathrm{sh}}-\tau_{1} \\ T_{\mathrm{sh}} & t_{\mathrm{sh}}-\tau_{1} \leq t \leq t_{\mathrm{sh}}+\tau_{2} \\ T_{\mathrm{FIT}}^{\mathrm{II}} & t \geq t_{\mathrm{sh}}+\tau_{2}\end{cases}$

where $T_{\mathrm{sh}}=\left(T_{\mathrm{FIT}}^{\mathrm{I}}\left(t_{\mathrm{sh}}\right)+T_{\mathrm{FIT}}^{\mathrm{II}}\left(t_{\mathrm{sh}}\right)\right) / 2$.

$t_{\mathrm{sh}} \sim 30 \mathrm{~min}$, the center time of the shoulder, is derived from the experimental data observations. The values of $\tau_{1}$ and $\tau_{2}$ result from the continuity conditions $T_{\mathrm{FIT}}^{I}\left(t_{\mathrm{sh}}-\tau_{1}\right)=$ $T_{\mathrm{sh}}\left(t_{\mathrm{sh}}-\tau_{1}\right)$ and $T_{\mathrm{FIT}}^{\mathrm{II}}\left(t_{\mathrm{sh}}+\tau_{2}\right)=T_{\mathrm{sh}}\left(t_{\mathrm{sh}}+\tau_{2}\right)$.
The fitted parameters for THEMIS B are given in Table 1. Furthermore we distinguish a penumbra region before the entry into eclipse and before re-entry into the sunlight; this part is not modelled, but it is taken care of by smoothly joining $T_{\mathrm{ESM}}$ with $T_{S}$ before and after the eclipse. The three lower panels in Fig. 2 illustrate the use of the original fake spin period (red curves) and of the model spin period (black curves) for despinning the magnetic field for the data of 7 March 2009. They contain the three components of the magnetic field in a despun spacecraft coordinate system where the $\mathrm{z}$-axis is aligned with the spin axis and the $\mathrm{x}-\mathrm{z}$ plane contains the direction toward the Sun. The lowest panel shows in blue the field magnitude that is very constant. This is a very good case for determining the spin periods from FGM data since the magnetic field has also an almost constant direction parallel to $\mathrm{x} ; B_{\mathrm{Y}}$ and $B_{\mathrm{Z}}$ are close to zero.

\subsection{The physics behind the fit function}

The fit function (2), which has been used to approximate the spin period behaviour in eclipse, is not an empirically found function. Instead the following physical considerations lead to its development. The spin period of the rotating spacecraft is proportional to the moment of inertia which is proportional to the square of the distance of the mass elements to the rotation axis. The temperature loss depends on the temperature of the spacecraft components conforming to Boltzmann's law and is proportional to the fourth power of the absolute temperature. The contraction in the radial direction is dominated by the contraction of the wire antennae and of the booms and depends linearly on the temperature loss. A few steps in the deduction of the fitting function are presented below.

When the spacecraft enters eclipse, the thermal budget will be mainly influenced by the emission of heat with a power proportional to $\theta^{4}$, where $\theta$ denotes its temperature. The heat energy content of the spacecraft will decrease with the rate proportional to the first derivative of $\theta$. Hence, the following differential equation will hold:

$c_{1} \theta^{4}=\frac{d \theta}{d t}$

$c_{1}$ is here an unknown constant, which includes the StefanBoltzmann-constant, the spacecraft's heat capacity, as well as a measure of its area to mass ratio. The differential equation can be easily solved by:

$\theta=\sqrt[3]{\frac{1}{c_{2} t+c_{3}}}$

Here $c_{2}$ is proportional to $c_{1}$ and $c_{3}$ denotes a constant of integration. Equation (5) describes the decrease in temperature of the spacecraft. If the wire boom length is assumed to behave proportionally to the temperature, the moment of inertia $I$ of the wire booms, which is basically proportional 
Table 1. Model parameters for THEMIS B.

\begin{tabular}{ccccc}
\hline$T_{\text {ESM }}$ & $a_{0}$ & $a_{1}$ & $a_{2}$ & $a_{3}$ \\
\hline$T_{\text {FIT }}^{\mathrm{I}}$ & $1.09102 \times 10^{-6}$ & $4.81989 \times 10^{-3}$ & $6.69644 \times 10^{-4}$ & 0.0 \\
$T_{\text {FIT }}^{\mathrm{II}}$ & $8.63622 \times 10^{-8}$ & $4.30367 \times 10^{-4}$ & $1.21247 \times 10^{-4}$ & $-3.67598 \times 10^{-3}$ \\
\hline
\end{tabular}

to the square of their length, should show the following time dependence:

$I=\sqrt[3]{\frac{1}{\left(c_{4} t+c_{5}\right)^{2}}}$

$c_{4}$ and $c_{5}$ are, again, unknown constants, which now also include a value for the mass of the wire booms and the constant of proportionality between temperature and wire boom length. Since the spacecraft conserves its angular momentum during the eclipse, the product $I \omega$ or the ratio $I / T$ should remain constant. Here $\omega$ and $T$ denote the angular spin frequency and spin period of the spacecraft respectively. Hence, the spin period behaviour should be analytically well described by the formula:

$T=\sqrt[3]{\frac{1}{\left(c_{6} t+c_{7}\right)^{2}}}$

where $c_{6}$ and $c_{7}$ also contain the angular momentum. For the computation of the spin period behaviour only the change in this period is considered. At $t=0$ (beginning of eclipse time) $\Delta T$ should be 0 . Hence, we added $-1 / \sqrt[3]{c_{7}^{2}}$ to Eq. (7). We also included to the final approximating function a scaling factor $\left(c_{8}\right)$ and a constant offset $\left(c_{9}\right)$, which is particularly important when the second branch (extended spin period model) is calculated, since the decay in period does not start from $T_{S \text { ref }}$ (corresponding to $\Delta T=0$ ) but from some shorter period (negative $\Delta T$ ). Thus we obtain the final fit function (2):

$\Delta T=\left(\sqrt[3]{\frac{1}{\left(c_{6} t+c_{7}\right)^{2}}}-\sqrt[3]{\frac{1}{c_{7}^{2}}}\right) c_{8}+c_{9}$

\section{Model check and error analysis}

The eclipse-spin model (ESM) derived in Sect. 2.3 can be applied for every eclipse without determining the spin period from the magnetic field. The ESM was checked against all eclipse data between March 2008 and July 2009. The final error $\phi$, an accumulated phase deviation, was estimated by adding up the spin periods during the eclipse and comparing with the first sun pulse time after the eclipse. The change of the period in the eclipse is approximately $0.005 \mathrm{~s}$ after $30 \mathrm{~min}$. This corresponds to 600 spins, since the THEMIS

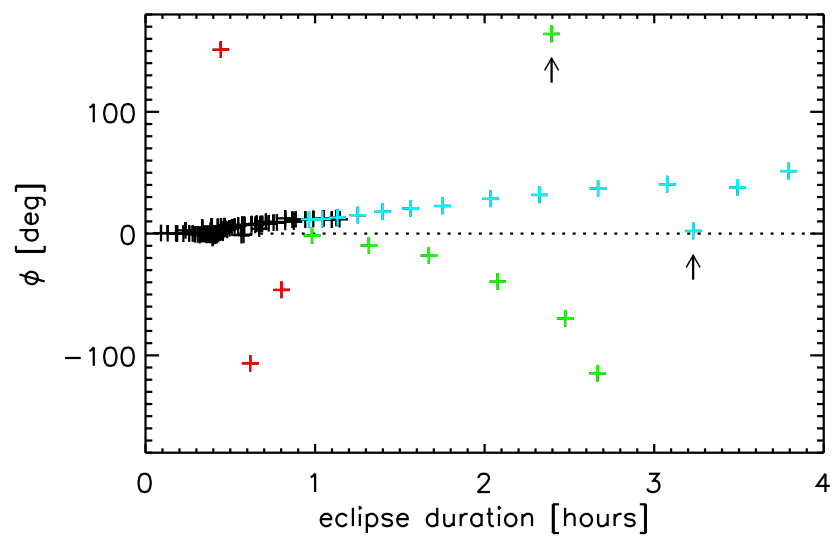

Fig. 3. Accumulated phase error, $\phi$ in degrees, versus eclipse length for the THEMIS B eclipses between March 2008 and July 2009. Black: all eclipses up to about $1 \mathrm{~h}$ duration for 2008 and 2009 , blue: long eclipses in 2009, green: long eclipses in 2008, red: bad results due to manoeuvres. The arrows point to the long eclipses with very long stay in penumbra.

spacecraft completes a rotation in $3 \mathrm{~s}$. Adding up the errors in the spin periods in the case when no correction is performed the Sun direction will be out of phase with one spin or $360^{\circ}$ after $30 \mathrm{~min}$. This is visible in the two middle panels of Fig. 2, where incorrectly despun $B_{\mathrm{X}}$ and $B_{\mathrm{Y}}$ (red lines) make an apparent 360 degrees rotation during the first $30 \mathrm{~min}$ and a total of 14 rotations during the $3 \mathrm{~h} 14 \mathrm{~min}$ of the eclipse. Figure 3 shows the errors in terms of phase deviation for the eclipses between March 2008 and June 2009 versus the duration of the eclipses. This interval has been chosen to cover the main phase orbits for THEMIS B, i.e. after the boom deployment and before the injection of the spacecraft into the transfer orbits for its journey to the moon. During these months the orbit of THEMIS B did not significantly change in shape, but rotated around the Earth due to the Earth's motion around the Sun; the tail seasons causing long eclipses are included for 2008 and 2009.

When applying the ES model to despin the magnetic field for the long eclipse (black curves in the two mid panels of Fig. 2) the accumulated phase error $\phi$ is less then $1^{\circ}$. The model was determined based on this case (blue cross marked with the black arrow on Fig. 3), so an error analysis of other applications of the model is of interest. 
Most of the eclipses do not last longer than one hour. The black crosses mark the eclipses close to perigee, i.e. in large magnetic fields. In these cases the modeled spin periods give us extraordinarily good results with accumulated phase deviations of less than 10 degrees. Short eclipses for which the model application was not successful are marked in red. In these three cases the spin period has been changed by the use of the spacecraft thrusters shortly before eclipse start. Hence, the reference spin period $T_{S \text { ref }}$ could not be obtained accurately. This results in a constant offset between the model spin period and true spin period which ultimately leads to a large accumulated phase deviation. The green crosses in Fig. 3 mark the long eclipses observed in March 2008 and the blue crosses those from March and April 2009. The accumulated phase deviation for 2009 is below 40 degrees for eclipses not longer than $3 \mathrm{~h}$. The same conclusion applies to the eclipses of 2008 not longer than $2 \mathrm{~h}$. An interesting feature of Fig. 3 is the increasing separation of the phase difference curve $\phi$ with increasing eclipse duration for the two years 2008 and 2009. Obviously, the second model branch, which has been determined from a 2009 eclipse, leads to better results for long eclipses of this year: The phase deviation increases drastically for 2008 eclipses longer than $2 \mathrm{~h}$. This means, that the spin period behavior was different in 2008 and 2009. One reason may be the fuel content of the spacecraft, which was larger in 2008. The release of fuel will probably have slightly changed the moment of inertial of the spacecraft over the course of time.

The crosses marked with arrows correspond to the first eclipses in March 2008 and 2009 of the series of eclipses marked in the two respective colors. The penumbra phase was particularly long in these cases, since the spacecraft only skimmed the region in full shadow and did not fly right through umbra.

From this statistical analysis we can conclude, that for short eclipses the accumulated phase error is below $10^{\circ}$ while it is up to $40^{\circ}$ for eclipses lasting less than $2 \mathrm{~h}$. A possibility to improve the application is to adapt the model to each individual eclipse. This can be done by distributing the phase deviation at the reentry in the sunlight over all the periods during the eclipse and reconstructing the sun-pulse times. The phase deviation after this adapted model application is close to zero (see Sect. 4).

\section{The effect of the Earth albedo radiation on the spin period}

Whenever the spacecraft comes close to the Earth, the infrared radiation form the Earth's surface becomes important for its thermal budget. Heating the spacecraft up increases its moment of inertia and, hence, its spin period. The radiation from Earth may be due to the reflection of sunlight (albedo) or simply thermal radiation of previously stored energy (on the night side). The Earth's radiation on the night side and the cooling of the spacecraft in eclipse have an opposite ef-

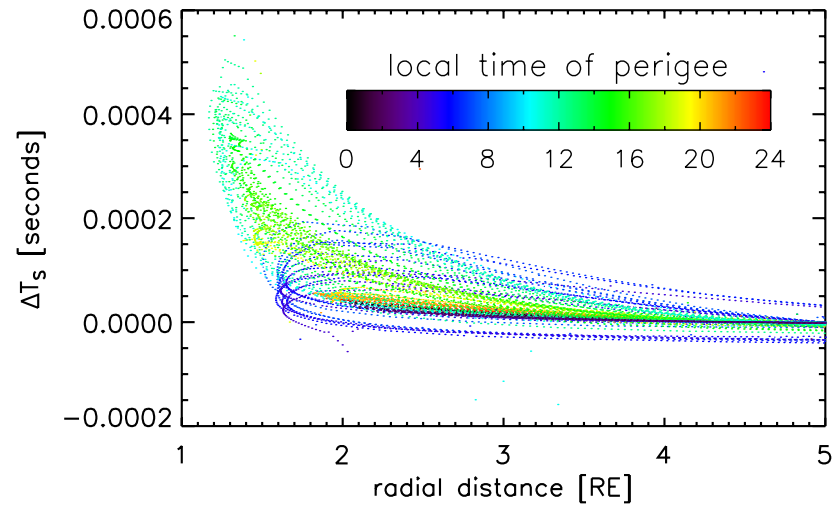

Fig. 4. Spin period change $\left(\Delta T_{S}\right)$ due to Earth radiation versus radial geocentric distance to the Earth. The colours show the local time (in hours) of the perigee crossing.

fect on the spin period behavior. Although it seems to be obvious, that in eclipse the spacecraft will overall cool down, the question remains how much slower this cooling process evolves in the presence of the additional terrestrial radiation.

In order to quantify this effect we performed the following statistical study of THEMIS B spin period data determined by the sun sensor from the interval March 2008 to July 2009. The interval was divided into subintervals including only times, at which the last eclipse had ended at least $12 \mathrm{~h}$ before and the next one lay at least $10 \mathrm{~min}$ in the future. This ensures that the spacecraft has had enough time to recover its natural spin period in thermodynamic equilibrium after eclipse, such that our analysis is not affected by the spacecraft cooling in shadow. For each of these subintervals the median of the spin periods of THEMIS B were computed belonging to times when the spacecraft was more than $5 R_{\mathrm{E}}$ (Earth radii) away from the center of Earth. These are the reference spin periods to which we can compare the ones measured closer to Earth. The change in spin period, $\Delta T_{S}$, with respect to these reference periods is shown for all the subintervals in Fig. 4.

As can be seen the effect is more important the closer the spacecraft approaches the terrestrial surface. For the minimum perigee distance to the surface of about $0.2 R_{\mathrm{E}}$ (corresponding to a radial distance to the center of the Earth of $1.2 R_{\mathrm{E}}$ ) the change in spin period can be at most $0.0005 \mathrm{~s}$. The effect is drastically diminished, when the spacecraft is further away from the Earth. For instance at $2.5 R_{\mathrm{E}}$ geocentric distance, $\Delta T_{S}$ does not exceed $0.0002 \mathrm{~s}$.

The color of the data points displays the local time position of the (temporally) closest perigee pass to the corresponding spin period considered. The closest approaches to the terrestrial surface correspond to the perigee passes around local noon (light blue and green dots), where we would expect the radiation from Earth to be largest due to the direct reflection of sun light in addition to the larger thermal radiation from the surface, which diminishes during night time. Both effects 


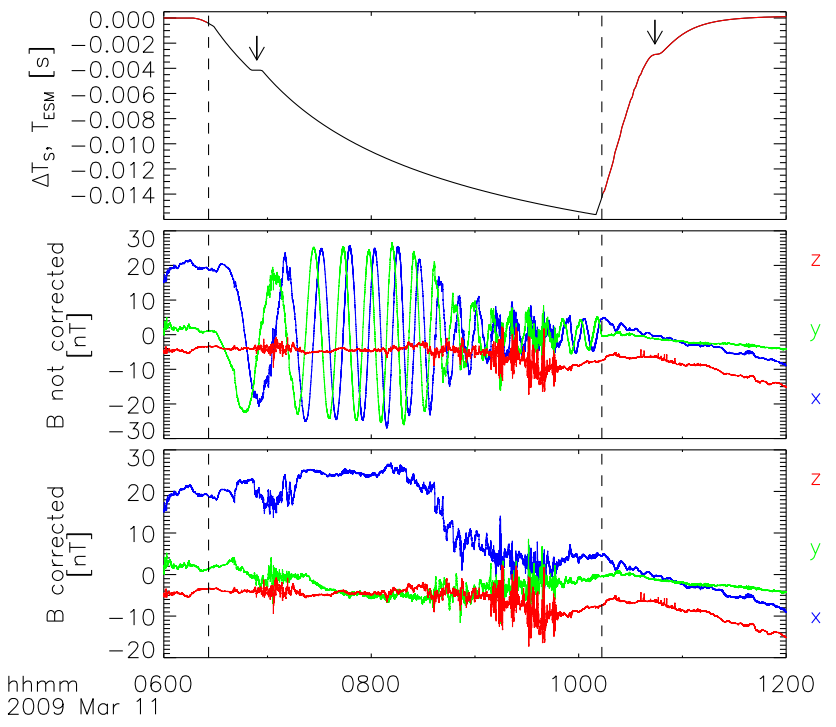

Fig. 5. ES model application to a long eclipse for THEMIS B on 11 March 2009. Top panel: spin period differences to $T_{S \text { ref }}$, mid panel: magnetic field despun with uncorrected spin times, bottom panel: magnetic field despun with corrected spin-times using the ESM. The eclipse time interval is delimited by the dashed lines.

(closer spacecraft and larger radiation) enhance the increase in spin period. Close-in perigee passes around local midnight are excluded from the analysis due to perigee in the umbra. Noon/afternoon (green dots) and midnight local time (red) passes can be compared at a distance of 2 RE from the Earth's center. The increase in the spin period produced by the Earth radiation is $<10^{-4} \mathrm{~s}$, whereas the decrease produced by the cooling in near-Earth eclipses is of the order of $5 \times 10^{-3} \mathrm{~s}$, so terrestrial radiation does not have an important contribution to the spin period change and has been neglected.

\section{Examples of model application}

\subsection{Application of the ES model in the Earth tail}

We illustrate in Fig. 5 the application of the ES model to the longest eclipse ( $3 \mathrm{~h} 45 \mathrm{~m}$ ) encountered by THEMIS B on 11 March 2009.

The red line in the top panel is, similar to Fig. 2, the difference of the spin period delivered by the sun sensor to the reference value prior to eclipse $\left(\Delta T_{S}\right)$. The limits of the eclipse [EStart, EEnd] are marked by the two vertical dashed lines. The thin black line is the ESM. The two lower panels show the 3 components of the magnetic field (x:blue, $\mathrm{y}$ :green, $\mathrm{z}$ :red) in a despun spacecraft frame, $\mathrm{z}$ aligned to the spin axis, $x$ toward the Sun. In the mid panel the fake constant value has been used for despinning while in the lower panel the model values ( $\left.T_{\mathrm{ESM}}\right)$ were used.

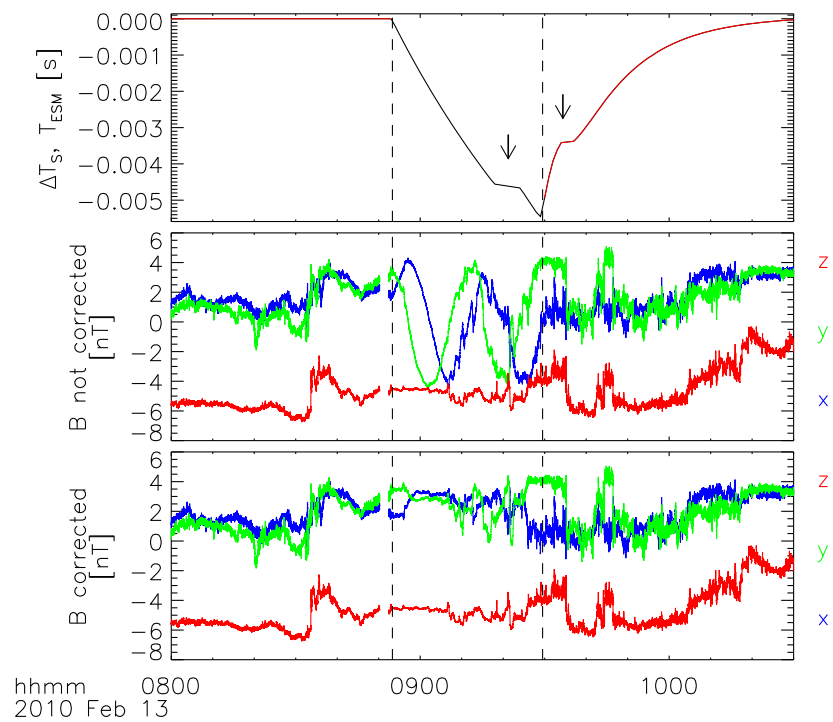

Fig. 6. ES model application for a THEMIS B lunar-flyby, 13 February 2010. Top panel: spin period differences to $T_{S \text { ref }}$, mid panel: Magnetic field despun with uncorrected spin times, bottom panel: magnetic field $\boldsymbol{B}$ despun with corrected spin-times using the ESM. The eclipse time interval is delimited by the dashed lines.

The modeled spin periods $T_{\mathrm{ESM}}$ are taken between $E$ Start and EEnd. EStart denotes the time $15 \mathrm{~s}$ prior to the eclipse onset, as flagged in the data, EEnd is the time of the eclipse end, from which time on sun sensor measurements are again available. The model $T_{\mathrm{ESM}}$ may be adapted to a particular eclipse by adding a small linear drift $\delta=d\left(T_{\mathrm{ESM}}\right) / d t$ yielding a modified spin period of $T_{\mathrm{ESM}}+\delta(t-E S t a r t)$. The free parameter $\delta$ will be used to reduce iteratively the final phase deviation to zero.

By using a linear interpolation between the last measured spin period and the first modeled one we ensure a smooth transition from the sun sensor measurements into the model. After EEnd the first $60 \mathrm{~s}$ of measured spin period data are linearly extrapolated to times before EEnd until the point, where this straight line extension and the modeled spin period function cross each other. Before this point in time the spin period is obtained from the model, afterwards the linear extrapolation bridges the last gap of eclipse time. This takes into account, that the spacecraft is heated up again shortly before $E E n d$ due to entering the penumbra region. Inside of this region sun sensor measurements are still not available, but the spin period increases again considerably. Furthermore, we ensure with the linear extrapolation a smooth transition from the model to the sun sensor measurements at eclipse end.

\subsection{Application of the ES model in the lunar wake}

The crucial question is to which extent the model can be applied to reconstruct the correct spin periods and sun pulse 
times during eclipses, where the true spin period and spacecraft rotation phase can no longer be derived from a terrestrial magnetic field model. Since two of the THEMIS spacecraft are flying to the moon to be inserted into lunarcentered stable orbits, we have had the unique opportunity to test the model with data from the recent lunar flyby of the THEMIS B spacecraft, which took place on 13 February 2010, see Fig. 6. The spacecraft entered lunar eclipse at about 08:53 UT. The end of the eclipse took place roughly 47 min later at about 09:30 UT. The duration of the eclipse was, hence, larger than the first branch of our model. Would this have been a terrestrial eclipse under the circumstances considered for our statistical analyses, a resulting phase deviation of the order of 10 degrees would have been expected.

The accumulated phase error is in this case 27.4 degrees, which is a low value in absolute terms, but quite a high value considering the length of the eclipse of only $47 \mathrm{~min}$. The explanation maybe the fuel consumption during the time elapsed since the eclipses used to define the model. After adapting the model to cancel out the final phase deviation we expect that the error in phase maximizes at the middle of the eclipse without exceeding half the value measured before adaptation. Although a minor deviation in phase may still be present in the despun data, the improvement is considerable: the model helped to recover the flyby data and make them usable for scientific purposes. A detailed study of solar wind transient features and the lunar wake structure during the flyby event based on a comparison of the eclipse-spin model despun data with results of hybrid simulations can be found in Wiehle et al. (2011).

\section{Discussion and conclusions}

The spacecraft spin behaviour during eclipse is characteristic for each spacecraft and reflects the dependence of its moment of inertia on temperature.

An "eclipse-spin model" for the spacecraft spin period behaviour can be developed by using the magnetic field vector measurements in a spinning frame. The magnetic field must be either close to Earth where its change in direction can be deduced from a model magnetic field or have a constant direction and a slowly varying magnitude in order to be usable for modelling. After defining an eclipse-spin model, it can be applied to eclipses in other regions of the magnetosphere or outside of it without the need of magnetic field measurements. The ES model compensates for the lack of experimental spin phase information from the sun sensor, such that satellite experiments, depending on correct spin phase information, can deliver science data even during eclipses.
The application of the method to CLUSTER and THEMIS data confirm the validity of the assumptions and give good results for the spin period reconstitution. This method can be applied to all spinning spacecraft carrying a vector magnetometer after a number of eclipses were recorded in the magnetosphere. The ESM defined for THEMIS will bring a valuable support to the ARTEMIS mission by the possibility to use the on-board instruments in the lunar shadow.

Acknowledgements. The authors thank Jim Lewis and the THEMIS software team for their invaluable help in providing the sun sensor and eclipse time data. The THEMIS mission is supported through NASA contract NAS5-02099; this work was financially supported by the German Ministerium für Wirtschaft und Technologie and the Deutsches Zentrum für Luft- und Raumfahrt under grant 50QP0402.

The service charges for this open access publication have been covered by the Max Planck Society.

Guest Editor M. Taylor thanks M. Dunlop and P.-A. Lindqvist for their help in evaluating this paper.

\section{References}

Auster, H. U., Glassmeier, K. H., Magnes, W., Aydogar, O., Baumjohann, W., D. Constantinescu, D., Fischer, D., Fornacon, K. H. , Georgescu, E., Harvey, P., Hillenmaier, O., Kroth, R., Ludlam, M., Narita, Y., Nakamura, R., Okrafka, K., Plaschke, F., Richter, I., Schwarzl, H., Stoll, B., Valavanoglou, A., and Wiedemann, M.: The THEMIS Fluxgate Magnetometer, Space Sci. Rev., 141(1-4), 235-264, doi:10.1007/s11214-008-9365-9, 2008.

Balogh, A., Carr, C. M., Acuña, M. H., Dunlop, M. W., Beek, T. J., Brown, P., Fornacon, K.-H., Georgescu, E., Glassmeier, K.H., Harris, J., Musmann, G., Oddy, T., and Schwingenschuh, K.: The Cluster Magnetic Field Investigation: overview of in-flight performance and initial results, Ann. Geophys., 19, 1207-1217, doi:10.5194/angeo-19-1207-2001, 2001.

Cherchas, D. B.: Dynamics of Spin- Stabilized Satellites during Extension of Long Flexible Booms, J. Spacecrafts and Rockets, 8(7), 802-804, 1971.

Lai, S. T., Mahon, H., and Smiddy, M.: Dynamics of Wire Boom Oscillations on a Spinning Satellite Part I, Lagrangian Equations of Motion and Transient Response, J. Appl. Math. Phys. (ZAMP), 30, 1-11, 1979.

Wiehle, S., Plaschke, F., Motschmann, U., Glassmeier, K.H., Auster, H. U., Angelopoulos, V., Mueller, J., Kriegel, H., Georgescu, E., Halekas, J., Sibeck, D. G., and McFadden, J. P.: First lunar wake passage of ARTEMIS: Discrimination of wake effects and solar wind fluctuations by 3D hybrid simulations, Planet. Space Sci., 59(8), 661-671, doi:10.1016/j.pss.2011.01.012, 2011. 